\title{
Dirección de comunicación y sector de telecomunicaciones: estudio de caso
}

\section{Communication management and telecom sector: a case study}

\author{
Mónica Recalde. Universidad de Navarra (mrecaldev@unav.es) \\ Elena Gutiérrez-García. Universidad de Navarra (egutierrez@unav.es)
}

Recibido: 21/10/2016 - Aceptado: 28/02/2017

\section{Resumen:}

Desde la academia, la comunicación y su papel en la gestión de las compañías continúa siendo objeto de intenso debate. Sin embargo, cuando se profundiza desde un ángulo estratégico, apenas se hallan análisis empíricos equiparables a otras realidades de gestión. Este estudio tiene como objetivo analizar la estructura y la actividad del departamento de comunicación de tres empresas de referencia en el sector de telecomunicaciones español: Telefónica, Vodafone y Orange. Para ello, se ha empleado una metodología cualitativa basada en cuestionarios y entrevistas en profundidad. Entre los resultados, se apunta a la complejidad de su organización, la importancia de la representación y participación en los órganos de dirección, así como a la influencia de la comunicación en la coordinación interdepartamental.

Palabras clave:

Comunicación; estrategia; gestión; telecomunicaciones; grupos de interés

\section{Abstract:}

From an academic perspective, the role of communication in company management remains a topic of intense debate. However, by delving deeper into the issue from a strategic point of view one can see that hardly any empirical analysis comparable to other management realities can be found. The aim of this study is to analyse the structure and activity of the communications departments in the three leading companies in the Spanish telecommunications sector: Telefónica, Vodafone and Orange. For that purpose, a qualitative methodology was used based on questionnaires and interviews. Among the results, some of the indicators are the complexity of the organisation, the relevance of representation and participation among the most important senior executives of the organization as well as the influence of communication on interdepartmental coordination.

Key words:

Communication; strategy; management; telecommunications; stakeholders 


\section{Introducción}

El contexto actual ha otorgado a la empresa un papel predominante, no sólo en la vida económica, sino también en la socio-política y cultural. Cada vez se acentúa más en foros académicos y profesionales su rol como institución social, se agranda la visión sobre su naturaleza y se amplifican los objetivos que debe contemplar en la esfera pública.

Precisamente, la importancia de los vínculos empresariales con los actores del entorno social (como por ejemplo entidades públicas, ONGs o representantes de comunidades locales) se ha convertido en uno de los aspectos más representativos de un nuevo enfoque de gestión empresarial. Directivos y gestores progresivamente toman sus decisiones, no de manera unilateral, sino considerando las ideas y demandas de distintas personas e instituciones, que se convierten en interlocutores partícipes y no meros receptores pasivos de información. Todo ello plantea a los profesionales numerosos retos sobre cómo administrar las demandas y expectativas de sus grupos de interés que, además, son cada vez más exigentes sobre la cantidad y calidad de información demandada (Herranz de la Casa, 2007).

Los investigadores sí vienen insistiendo desde final del siglo XX en cuestiones que atañen a la buena marcha empresarial y el papel que juega la disciplina en dicha ecuación: ¿Cómo gestionar las relaciones con múltiples stakeholders? ¿Cómo responder a las demandas de sostenibilidad y responsabilidad? ¿Cómo recuperar y/o cultivar la confianza en el mundo de los negocios? Sobre estas y otras cuestiones, por su parte, varias escuelas de Management lo enfocan desde diversas ramas teóricas: stakeholder management, Responsabilidad Social Empresarial, gobierno corporativo, etc.

Pero el objetivo de este artículo no es centrarse en estas abundantes perspectivas, sino más bien estrechar lazos entre la comunidad académica y profesional sobre el papel de la Dirección de Comunicación en las compañías. Con tal fin, la estructura del trabajo es la siguiente. En primer lugar, se presenta un marco teórico centrado en la revisión de las funciones del departamento de comunicación, el rol de su responsable en la alta dirección y, por último, la coordinación con otras áreas. Estos tres ejes se emplean como ancla para analizar el caso de tres grandes empresas multinacionales del sector de las telecomunicaciones: Telefónica, Vodafone y Orange. El trabajo termina con la discusión, y las conclusiones abren la puerta a los retos de las direcciones de comunicación en el futuro.

\subsection{Gestión estratégica de la comunicación: análisis holístico}

Buena parte de la literatura sobre comunicación corporativa ha atribuido a la comunicación una dimensión estratégica dentro de las organizaciones, enfoque que no ha hecho sino reforzarse en años recientes (Miquel y Navarro, 2016; Recalde y Gutiérrez-García, 2015; Berger y Meng, 2014; Falkheimer, 2014; Gregory y Willis, 2013; Van Riel, 2012).

Para Falkheimer (2014: 130) "la comunicación estratégica está relacionada con la importancia del mantenimiento, el cambio o el fortalecimiento de las imágenes de la organización entre sus grupos de interés". Cornelissen (2004: 5) define la 
comunicación corporativa como "una función de gestión que se encarga de la supervisión y coordinación de la labor realizada por profesionales de la comunicación especializados en disciplinas tales como relaciones con los medios, relaciones públicas y comunicación interna". En la misma línea, Falkheimer et al. (2016: 153) afirman que "existe una fuerte creencia en la comunicación como una función de gestión, y en sus profesionales por hacer todo lo posible por integrarla como una dimensión natural de la racionalidad de gestión”.

Precisamente esta gestión mira hacia un entorno multistakeholder. Freeman (1984) desarrolla esta idea en la teoría de los grupos de interés o stakeholders. En ella amplía el foco corporativo, previamente limitado a los propietarios o accionistas (shareholders) a todos aquellos grupos que se ven afectados o pueden afectar el desarrollo de la actividad de la organización (stakeholders). Ejemplos de ellos son: clientes, empleados, proveedores, instituciones públicas, ONGs, comunidades locales, etc.

Dentro de este contexto, un análisis del estado de la cuestión -en la academia y en los informes profesionales-permite reconocer tres grandes áreas de investigación en la dirección de comunicación. En primer lugar, aquélla que estudia cómo se armonizan las distintas funciones en los departamentos de comunicación-marketing, publicidad, relaciones públicas, gabinete de prensa, etc.-. En segundo, la relación entre el denominado director de comunicación -más conocido por su acrónimo dircom- y la alta dirección o comité ejecutivo. Y, finalmente, la coordinación y los puentes entre el departamento de comunicación con otras unidades estratégicas de la empresa.

Estas tres grandes ramas brotan de un tronco común, el que responde a una visión holística y estratégica de la comunicación, tal y como lo expresan Christensen et al., (2008: 3): "La comunicación corporativa se basa en la metáfora del holismo: el deseo de abarcar todo dentro de una perspectiva o marco". En la siguiente figura se expone un marco teórico original que aúna tres grandes áreas fragmentadas en la academia bajo un paraguas común. Para hacer posible la mencionada gestión holística, la dirección de comunicación se rige bajo los principios de integración, alineación y forma parte del comité directivo de la empresa. 
Cuadro 1: Marco teórico: gestión holística y estratégica de la función de comunicación

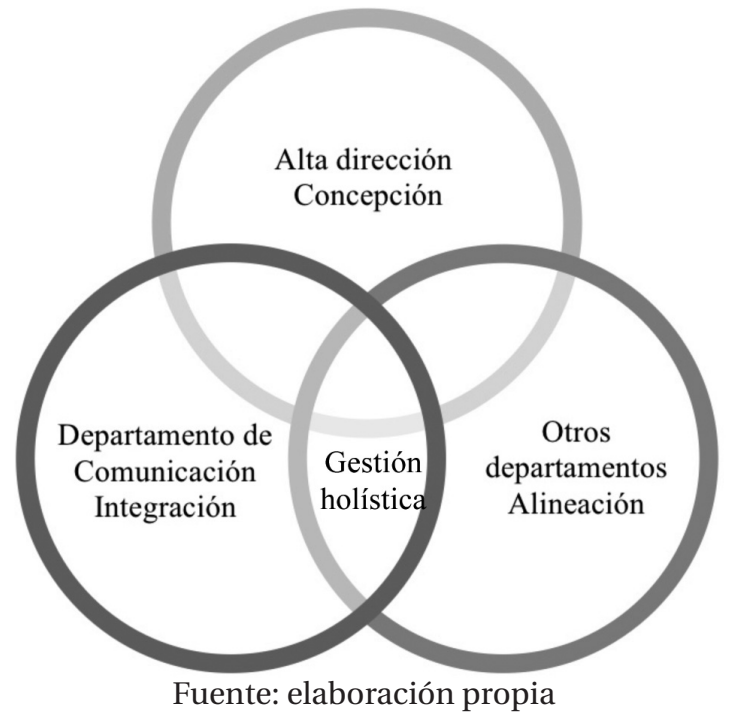

A continuación, se desarrolla cada uno de los puntos de este marco teórico para, posteriormente, presentar el caso de estudio que lo ilustra.

\subsubsection{Departamento de comunicación-integración}

La existencia de un departamento, división o unidad autónoma dedicada a la actividad comunicativa revela la importancia que los directivos pueden otorgar a dicha labor (Blay, Benlloch y Sanahuja, 2014). Desde la literatura se plantea que dicha función ha de estar guiada por el principio de integración. Así la definen como "el concepto y la práctica de la alineación de símbolos, mensajes, procedimientos y comportamientos para que una organización pueda comunicarse con consistencia, coherencia, claridad y continuidad dentro y a través de las fronteras organizacionales formales" (Christensen et al., 2008: 424).

Tomando estas palabras, la comunicación integrada no es únicamente la difusión de los mensajes en todos los soportes y medios, sino el hecho de responder a una visión coherente de la identidad de la organización (Costa, 2011; Losada Vázquez, 1998). Por esta razón, su esencia reside en organizar la actividad según una coordinación de equipos y gestión integral bajo la batuta de un máximo responsable que vigile la aplicación efectiva, en este caso el dircom.

Si bien puede haber en cada organización más de una unidad dedicada a comunicación existen modos de coordinarlas en una estructura coherente que evite solapamientos e inconsistencias (Argenti, 2014; Cornelissen y Lock, 2001; Van Riel,

80 | n² 24, pp. 77-101 | doxa.comunicación 
1997). Como cualquier otra área en la empresa, para desarrollar las distintas funciones y actividades se precisa de un conjunto de profesionales con distintos perfiles, desde técnicos a directivos.

En la última década, distintas investigaciones han encontrado importantes hallazgos empíricos sobre el estado de la profesión ofreciendo, al mismo tiempo, una radiografía de la situación particular de cada país (Gregory, 2011; Swerling y Sen, 2009). Concretamente, el European Communication Monitor recoge las respuestas de 2710 profesionales de la comunicación en 43 países. En su trabajo Zerfass et al (2016) aportan datos longitudinales (2007-2016) que revelan grandes cambios en la gestión de la comunicación, los canales y las necesidades de formación del personal implicado en las organizaciones.

También en el caso español, la Asociación de Directivos de Comunicación (Dircom) publica regularmente estudios acerca de la formación, competencias y responsabilidades del profesional de la comunicación, así como su implicación y participación en el comité de dirección (Dircom, 2016, 2015, 2014, 2010, 2005). Un estudio de 2015 desvela que más del $80 \%$ de los encuestados estima que la comunicación en sus organizaciones ha incrementado en importancia. Además, para el $74 \%$ la estrategia de comunicación contribuye bastante o mucho a la estrategia global de la compañía (Dircom, 2015).

\subsubsection{Alta dirección-concepción}

Distintos autores y la profesión han incidido en la necesidad de legitimar la figura de un directivo que ejerza con plena autoridad en los órganos de la alta dirección, el denominado dircom (Gutiérrez-García, 2014; Johansson y Ottesting, 2011; Gregory, 2008, González y Monleón, 2013). Precisamente, tratan de explicar el grado de relación con la alta dirección (Swerling y Sen, 2009; Grunig et al., 2002; Moss et al., 2000). Entre sus responsabilidades, se encuentran asesorar al comité directivo, por ejemplo, sobre la identificación de asuntos o temas sociales emergentes estratégicos, los efectos de las decisiones sobre la reputación o el desarrollo del discurso institucional. Además, ayudan a trasladar a los niveles directivos las demandas y expectativas de los públicos para una mejor toma de decisiones (Gutiérrez-García y Recalde, 2016; Frandsen y Johansson, 2015; Swerling y Sen, 2009).

Sin embargo, algunas investigaciones empíricas revelan ciertas barreras o dificultades que impiden que los altos directivos abran las puertas al director de comunicación como una figura importante de gestión (Gutiérrez-García, 2014; Brønn, 2014; Cornelissen y Thorpe, 2001; Moss et al., 2000). Entre ellas, la relativa juventud de la profesión, su limitada formación directiva, o la escasa comprensión de los altos directivos sobre el papel transformador de la comunicación, más allá de un rol táctico (Arthur W. Page Society, 2007: 1).

De ahí que, de acuerdo a Swerling y Sen (2009: 143), si los dircom reportan a la alta dirección, “los profesionales de la comunicación deben estar involucrados en reuniones estratégicas, sus recomendaciones tendrán que ser tomadas en serio para contribuir a la toma estratégica de decisiones". De acuerdo con los datos aportados por Dircom (2015), el 76 \% de los 
dircom depende del primer nivel ejecutivo, porcentaje que viene incrementándose paulatinamente desde 2005. Así, el $32,03 \%$ reporta directamente al director general, un 29,87 \% trabaja bajo supervisión del presidente y un 15,58 \% depende del consejero delegado.

No obstante, algunas publicaciones matizan que rendir cuentas a la gerencia no garantiza, necesariamente, que el dircom participe en las mesas directivas o tenga voto en la toma de decisiones. De hecho, puntualizan una diferencia, nada baladí, entre participar activamente en la toma de decisiones de la alta dirección y simplemente estar presente cuando las decisiones se toman (Moss et al. 2000).

\subsubsection{Otros departamentos-alineación}

Para comprender la verdadera naturaleza comunicativa, Cornelissen et al. (2006) la consideran una función transversal -también denominada cross functional o cross disciplinary-. Es decir, más allá de la existencia de un departamento de comunicación con competencias propias y exclusivas, es importante que ejerza una función colaborativa, de apoyo y que promueva sinergias gerenciales.

De ahí que la unidad de comunicación no pueda actuar en solitario, sino que presta servicio a toda la compañía porque es la sede del trabajo profesional organizado y planificado. Además, coordina las múltiples relaciones con stakeholders, así como las actividades, productos y servicios informativos.

Por esta razón, los sistemas internos para alinear la unidad de comunicación con el resto de departamentos son una pieza clave del puzle. Comités permanentes, reuniones periódicas, diseño de estudios conjuntos, entre otros, pueden reportar información valiosa al departamento de comunicación y al resto de áreas. Al mismo tiempo, quienes trabajan en él deben tener una visión abierta y no centralizadora, entendiendo que la comunicación compete a toda la organización e implica una cultura relacional compartida.

A continuación, se presenta un caso de estudio paradigmático que ahonda en el marco teórico presentado. Éste nos permitirá contrastar en la realidad cómo se gestionan las unidades dedicadas a la comunicación y su labor estratégica en empresas de telecomunicaciones.

\section{Metodología}

\subsection{Marco de estudio: gestión comunicativa en las empresas de telecomunicaciones}

Las empresas de telecomunicaciones y su amplia oferta tienen gran relevancia en el desarrollo socioeconómico de los países e impactan en sectores tan estratégicos como la administración pública, la educación o la sanidad. La importancia 
de la comunicación es consustancial a una industria de alta penetración en el mercado, sometida a una intensa vigilancia regulatoria y a los vaivenes tecnológicos. Las operadoras se convierten en destinatarias de información porque reciben multitud de mensajes del entorno pero, al mismo tiempo, reportan información sobre su actividad, oferta comercial y valores institucionales. Por ello establece y mantiene relaciones con un gran abanico de stakeholders y, en mayor o menor grado, aplicando muchos de los principios e ideas analizadas en la primera parte de este artículo.

Sin embargo, la mayor parte de la investigación sobre el sector en materia de comunicación hace referencia a la dimensión comercial, más concretamente en lo que respecta al consumidor (Cambra et al., 2012) y a los factores de fidelización (Wang, 2009). Pero existe un interés más allá de la comunicación comercial para adentrarse en una perspectiva corporativa. Como muestra, el caso de estudio de la comunicación interna en distintas operadoras, sobre todo en lo referido a las relaciones entre la matriz y sus filiales territoriales (Caballero, 2002) o el referente a la imagen y reputación de los operadores móviles (García de los Salmones, 2001). Los autores coinciden en señalar la imagen de la empresa como factor determinante en la elección del operador y subrayan que "la imagen necesita de una sustancia real, así gestionar la identidad es gestionar la imagen pero no se puede invertir el proceso" (Walesska y Alvarado, 2009: 13).

En este marco, existen escasos precedentes que analicen cómo se gestiona la comunicación estratégica en este sector: el estudio de las funciones del departamento de comunicación, los puentes que se trazan con otras áreas de la empresa, así como el impacto de la función comunicativa y de su responsable en los órganos de alta dirección. En otras palabras, la siguiente investigación presenta unos hallazgos originales dado que ahondan en un sector que, aun resultando clave, ha sido poco explorado en los estudios de comunicación corporativa.

\subsection{Un estudio sobre la comunicación estratégica en telecomunicaciones}

El presente trabajo se plantea, como objetivo general, analizar la estructura y la actividad del departamento de tres operadoras líderes en España: Telefónica, Vodafone y Orange. En concreto, se parte de la hipótesis de que estas empresas realizan una gestión estratégica de su comunicación en un entorno multistakeholder. Siguiendo con el esquema del marco teórico se persiguen tres objetivos: 1) estudiar el departamento de comunicación: sus divisiones y funciones, 2) qué representación tiene en los órganos de dirección y 3) de qué manera se coordina con otras áreas estratégicas.

Los objetivos de investigación y su naturaleza cualitativa demandan un tipo de muestreo no probabilístico. De hecho, la selección de tres empresas -Telefónica, Vodafone y Orange- responde a un muestreo teórico, no estadístico, que busca una controlada elección de los sujetos de la muestra (Denzin y Lincoln, 1994).

Esta selección se justifica en términos de relevancia y homogeneidad. El último informe anual publicado por la Comisión Nacional de los Mercados y de la Competencia de las telecomunicaciones revela el peso de estas compañías en su sector. 
Como muestra, en telefonía fija las tres copan el 92,5\% del mercado. Mientras que Movistar tiene un 51,7\% del total de líneas, Vodafone y Orange (con Ono y Jazztel incluidos respectivamente) suma el $41 \%$ del total de líneas, repartido a partes iguales. De la misma manera, en el caso de la telefonía móvil, las empresas elegidas lideran en cuota de líneas con un 30,7\% Movistar; 26,9\% Orange y 25,3\% Vodafone en (Comisión Nacional de los Mercados y de la Competencia, 2016).

Junto al criterio de relevancia, todas ellas son multinacionales con unas estructuras y recursos que permiten cierta homogeneidad para llevar a cabo un estudio comparativo: tienen una oferta semejante de productos y servicios -telefonía fija, móvil, banda ancha y señal audiovisual-, una organización multinacional y, por último, una estructura consolidada del departamento de comunicación.

Unido a ello, los profesionales de la muestra debían contar con un gran rango de conocimiento, aunar una visión estratégica y ejecutiva, haber tenido experiencia en las distintas divisiones del departamento de comunicación y, por último, contar con la autoridad suficiente para responder en nombre de la organización.

Los autores de la investigación, siguiendo un acuerdo de confidencialidad, revelarán los resultados aunque no aportarán datos individuales de cada uno de los profesionales de la muestra. En el siguiente cuadro se aporta información conjunta del perfil profesional seleccionado, su experiencia y conocimiento, el tiempo de experiencia en sus puestos, la experiencia previa en la empresa así como sus responsabilidades. Toda esta información se presenta de manera acumulada y anónima.

Cuadro 2. Características de la muestra

\begin{tabular}{|c|c|c|c|c|}
\hline PERFIL PROFESIONAL & $\begin{array}{l}\text { EXPERIENCIA Y CONO- } \\
\text { CIMIENTO }\end{array}$ & $\begin{array}{l}\text { TIEMPO EN } \\
\text { SUS PUESTOS }\end{array}$ & $\begin{array}{l}\text { ÁREAS DE EXPERIEN- } \\
\text { CIA PREVIA EN LA } \\
\text { EMPRESA }\end{array}$ & $\begin{array}{l}\text { RESPONSABILIDADES } \\
\text { ESPECÍFICAS }\end{array}$ \\
\hline $\begin{array}{l}\text { Ejecutivos de Comuni- } \\
\text { cación (Mandos } \\
\text { intermedios, } 2^{\circ} \text { nivel en } \\
\text { el departamento de Co- } \\
\text { municación ) }\end{array}$ & $\begin{array}{l}\text { Marketing, relaciones } \\
\text { públicas y comunica- } \\
\text { ción, fuerte } \\
\text { conocimiento y entendi- } \\
\text { miento de nuevas } \\
\text { tendencias }\end{array}$ & (3-8 años) & $\begin{array}{l}\text { Comunicación interna y } \\
\text { externa, comunicación } \\
\text { digital, marketing \& pu- } \\
\text { blicidad, gestión de la } \\
\text { reputación }\end{array}$ & $\begin{array}{l}\text { Coordinación de planes } \\
\text { de comunicación, orga- } \\
\text { nización de eventos, } \\
\text { relaciones con medios, } \\
\text { documentación e inves- } \\
\text { tigación }\end{array}$ \\
\hline
\end{tabular}

Fuente: elaboración propia

Para analizar la estructura y actividad del departamento de comunicación se empleó una metodología cualitativa (Maxwell, 1996). La necesidad de estudiar la complejidad de los fenómenos organizativos con un objetivo de comprensión más 
descriptivo que de búsqueda de relaciones causales justifica la aplicación de esta metodología en Ciencias Sociales (Patton, 1990). En las siguientes líneas se detallan los métodos empleados:

-Cuestionarios auto-administrados: en una primera fase del trabajo de campo se envió un cuestionario a cada una de las empresas, destinados a un profesional del departamento de comunicación previamente seleccionado. Este cuestionario consta de 19 preguntas de distinto tipo: abiertas, de respuesta múltiple y de valoración de escala Likert.

-Entrevistas en profundidad semiestructuradas: una vez analizados los resultados de estos cuestionarios en una segunda fase se realizaron entrevistas en profundidad a estos tres profesionales. Estas constaron de 13 preguntas, tuvieron lugar en Madrid y su duración osciló entre los 30 y los 45 minutos. Este doble método, pactado desde el principio con los sujetos de la muestra, se justifica porque la formulación de algunas preguntas como aquellas relacionadas con las cadenas de mando en el organigrama y los criterios en la toma de decisiones precisan respuestas más amplias a las de un cuestionario.

Cuadro 3. Guiones cuestionario y entrevistas

\begin{tabular}{|c|c|}
\hline Cuestionario & Entrevistas \\
\hline $\begin{array}{l}\text { 1-Departamento y competencias profesionales } \\
\text { - Coordinación interdepartamental } \\
\text { - Actividades que dirige y gestiona } \\
\text { - Control presupuestario }\end{array}$ & $\begin{array}{l}\text { 1-Organigrama } \\
\text { - Ubicación en el organigrama } \\
\text { - Reporte de información } \\
\text { - Perfil directivo y comité directivo } \\
\text { - Formulación de la estrategia comunicativa }\end{array}$ \\
\hline $\begin{array}{l}\text { 2-Relaciones con los públicos } \\
\text { - Identificación } \\
\text { - Valoración de cualidades } \\
\text { - Programas de comunicación y públicos } \\
\text { - Opinión profesional sobre la actividad comunicativa }\end{array}$ & $\begin{array}{l}\text { 2-Fundamentos de la comunicación } \\
\text { - Identidad, misión y cultura corporativas } \\
\text { - Propiedades de la comunicación en el sector de telecomu- } \\
\text { nicaciones }\end{array}$ \\
\hline $\begin{array}{l}\text { 3-Rol de la comunicación en la toma de decisiones } \\
\text { - Perfiles profesionales } \\
\text { - Funciones } \\
\text { - Representación directiva } \\
\text { - Herramientas y periodicidad de investigación } \\
\text { - Autonomía profesional }\end{array}$ & $\begin{array}{l}\text { 3- Estrategia } \\
\text { - Alineación con los objetivos estratégicos } \\
\text { - Estimulación de la innovación } \\
\text { - Sistemas de evaluación }\end{array}$ \\
\hline
\end{tabular}




\begin{tabular}{|l|l|}
\hline \multicolumn{1}{|c|}{ Cuestionario } & \multicolumn{1}{c|}{ Entrevistas } \\
\hline $\begin{array}{l}\text { 4- Perfil profesional } \\
\bullet \text { Formación }\end{array}$ & $\begin{array}{l}\text { 4-Gestión de relaciones } \\
\text { - Monitorización del entorno y tendencias } \\
\bullet \text { Experiencia profesional }\end{array}$ \\
\hline
\end{tabular}

Fuente: elaboración propia

Los investigadores analizaron los cuestionarios y entrevistas utilizando un método inductivo basado en tres categorías de contenido con diferentes subcategorías.

- Departamento de Comunicación: estructura del departamento o divisiones, tipo de coordinación entre las divisiones, cuáles son sus funciones principales.

- $\quad$ Relaciones con la alta dirección: cuáles son las funciones principales del dircom, a quién reporta esta figura, si forma parte o no del comité directivo, si participa o no en la toma de decisiones de este órgano.

- Coordinación del departamento de comunicación con otros departamentos: coordinación entre filiales y la empresa matriz, con qué áreas se relaciona el departamento de comunicación, con qué frecuencia, qué herramientas emplean para dicha coordinación.

\section{Resultados}

Las siguientes líneas recogen un rico debate presente en la academia y la profesión sobre el papel del departamento de comunicación dentro de las compañías en España. Siguiendo el orden presentado en la revisión teórica se desglosan los resultados en tres áreas: departamento de comunicación e integración, la alta dirección y el dircom y la alineación con otros departamentos.

\subsection{Gestión estratégica de la comunicación: análisis holístico}

Este epígrafe expone cómo se elaboran los planes de comunicación y en qué medida dichos planes se vinculan con los objetivos estratégicos de la compañía.

La Dirección de Comunicación de Vodafone entiende la comunicación como una actividad estratégica y de gestión dentro de la empresa. Por este motivo, especifican que "además de las operaciones rutinarias necesarias -gabinete de prensa, difusión de información o comunicación con empleados-, participamos directamente en procesos de planificación estratégicos" (Vodafone). 
También elaboran sus planes de manera autónoma. Sí, en cambio, acostumbran a consultar de manera informal algunos programas de comunicación con otros departamentos como las Unidades de Negocio o Recursos Humanos. Dicha tarea se considera prudente en los distintos procesos operativos. En este sentido, se sienten responsables de asesorar a otras unidades en materia de comunicación, por ejemplo, en la realización de comunicados públicos y elaboración de mensajes.

Entre sus fines, destacan, por encima de los demás, servir a los objetivos comerciales de la empresa dado que "los objetivos de comunicación se vinculan directivamente a los objetivos estratégicos de negocio por lo que trabajamos en común con las Unidades de Negocio" (Vodafone).

En Telefónica, igual que en el caso de Vodafone, se subraya "la comunicación tiene una consideración estratégica y está alineada con los objetivos comerciales de la empresa” (Telefónica). Es más, al igual que en Vodafone, cuando formulan los objetivos de comunicación los vinculan directamente con los objetivos estratégicos de la empresa -cuota de mercado, facturación, concesión de licencias, etc.-. También la Dirección de Comunicación goza de suficiente autonomía en la elaboración de planes estratégicos y, al contrario, que Vodafone, no consultan de manera informal sus programas con otros departamentos.

En Orange, la labor del departamento de comunicación se define como soporte del trabajo de otras unidades: "En realidad lo que nosotros hacemos es "traducir" a un lenguaje asequible para el público lo que ellos hacen, destacando lo que consideramos más relevante e interesante” (Orange).

Sobre los valores estratégicos la responsable entrevistada expone:

“En nuestras manos está la imagen de la empresa, el valor de la marca dado que es una de las más valoradas a nivel mundial, la reputación de nuestra empresa. Influimos también en la actividad comercial porque al final nosotros contribuimos con nuestro trabajo y formamos parte de un equipo de comunicación que es publicidad, vendemos los productos de nuestra empresa así como los resultados de nuestra compañía. En este sentido, también es importante saber cuándo, dónde y cómo deben ser comunicados. Además, mantenemos la transparencia como uno de los valores de la compañía. Es evidente que no en todos los momentos comunicas todo pero tratamos de ser todo lo más transparentes posibles. Porque la transparencia repercute en la confianza y esto vale tanto para el cliente como para un periodista" (Orange).

Precisamente, esta afirmación subraya el entorno multistakeholder citado en la primera parte de este trabajo. Los planes de comunicación en las tres empresas del estudio contemplan la identificación de grupos de interés estratégicos, así como los temas relevantes para ellos.

Sin embargo, a pesar de manifestar la importancia de la gestión estratégica, en Orange explican las dificultades con las que habitualmente se encuentran para planificar a largo plazo en las áreas de comunicación. En este sentido, destacan que: 
"Hacer un plan anual de comunicación es como lanzar la carta a los reyes magos. La comunicación la vamos haciendo de acuerdo a los planes de la empresa y, según van surgiendo distintos proyectos, se van estableciendo estrategias de comunicación. Estrategias de comunicación que pueden ser proactivas o reactivas porque no todas las cosas interesa comunicarlas de forma proactiva. Pero planes de comunicación, ya me gustaría a mí hacer un plan de comunicación a largo plazo" (Orange).

Los hallazgos hasta aquí descritos, muestran que los tres profesionales entrevistados se enfrentan a las dificultades de planificar a medio plazo pues, argumentan, el sector se caracteriza por el cambio continuo y, en consecuencia, la comunicación debe adaptarse a los vaivenes del entorno tecnológico, económico y social: "El sector de telecomunicaciones es tan dinámico que lo que vale para este mes a lo mejor para el mes siguiente ya no vale. Entonces la comunicación tiene que ser igual de ágil" (Orange). También hay unanimidad entre los tres responsables cuando manifiestan que la comunicación ha de ser entendida como una actividad formalizada, integrada en los procesos de gestión y de gobierno de la compañía. Además, añaden que debe estar indisolublemente unida a los planes estratégicos de sus compañías y, en consecuencia, a los objetivos comerciales y corporativos.

\subsubsection{Objetivo de investigación 1: departamento de comunicación}

Como apuntan ciertos autores, la organización interna tiene un impacto indudable en el modo en que se ejerce la función comunicativa (Christensen et al., 2008). Los siguientes resultados muestran la estructura del departamento, el conjunto de funciones y la coordinación interna.

\section{Estructura}

Respecto a la estructura ${ }^{1}$, el propósito de los siguientes cuadros no es mostrar los organigramas organizacionales sino ilustrar las divisiones o áreas que componen los departamentos de comunicación de las empresas en línea con los objetivos de esta investigación. Telefónica difiere de las otras dos compañías, dado que no existe una filial española, pues Telefónica es la matriz multinacional española. La figura muestra la estructura de la Dirección de Comunicación Corporativa y Medios de todo el grupo.

1 Las estructuras de las direcciones de las tres compañías descritas han podido sufrir variaciones cuando se difundan los resultados de este estudio. 
Cuadro 4. Dirección de Comunicación Corporativa y Medios de Grupo Telefónica

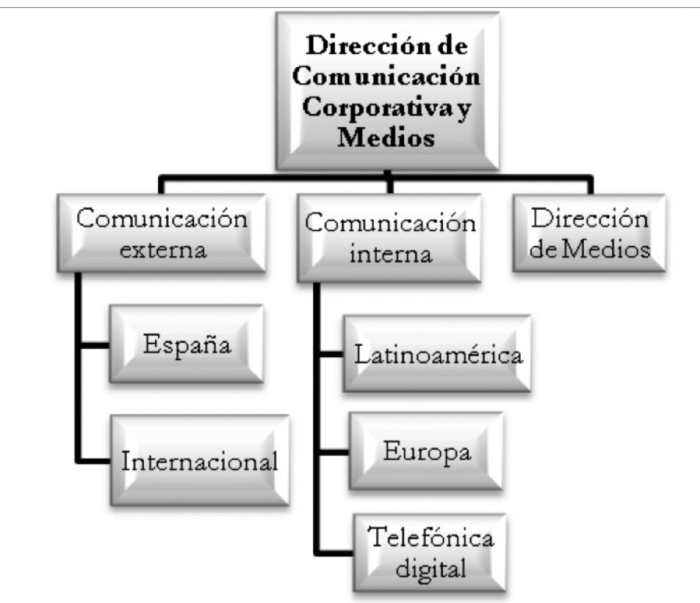

Fuente: elaboración propia a partir de entrevistas

Por su parte, Vodafone España organiza la comunicación en una única dirección denominada Dirección de Comunicación y Sostenibilidad. Ésta se subdivide, a su vez, en cuatro según puede verse en la siguiente figura.

Cuadro 5. Dirección de Comunicación y Sostenibilidad de Vodafone España

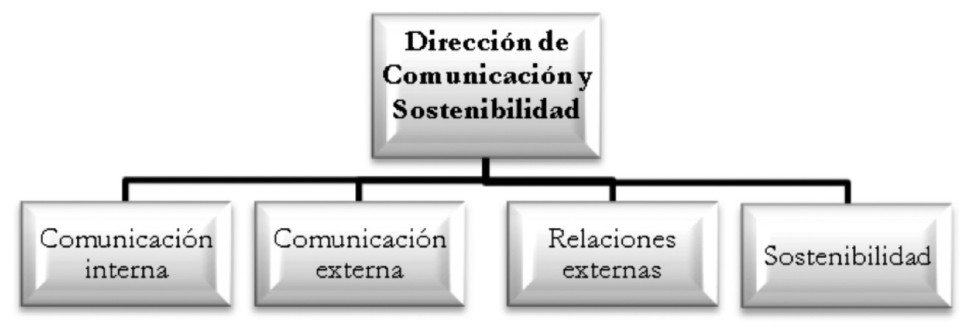

Fuente: elaboración propia a partir de entrevistas en profundidad

El departamento de comunicación de la filial española de France Télécom Orange, recibe el nombre de Dirección General de Comunicación y Marca. 
Cuadro 6. Dirección General de Comunicación y Marca Orange España

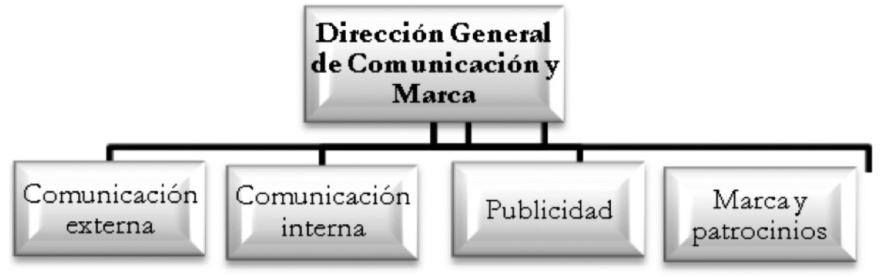

Fuente: elaboración propia a partir de entrevistas en profundidad

Una atenta mirada hacia las estructuras representadas permite destacar que las tres compañías cuentan con una dirección propia de comunicación, con unas competencias propias y es sede del trabajo profesional organizado.

\section{Funciones del departamento}

Si se atiende a la actividad de cada uno de estos departamentos, puede observarse que gestionan y desarrollan distintas funciones. Se entiende por funciones el conjunto de competencias que la unidad de comunicación realiza de manera habitual. La siguiente tabla resume los resultados globales relativos a esta cuestión.

Cuadro 7. Funciones de los departamentos de comunicación

\begin{tabular}{|l|c|c|c|}
\hline FUNCIONES & Vodafone & Telefónica & Orange \\
\hline Comunicación interna & $\bullet$ & $\bullet$ & $\bullet$ \\
\hline Relaciones con los medios & $\bullet$ & $\bullet$ & $\bullet$ \\
\hline Contenido online & & $\bullet$ & $\bullet$ \\
\hline Relaciones institucionales & & & \\
\hline Publicidad y marketing & $\bullet$ & $\bullet$ & \\
\hline Documentación e investigación & $\bullet$ & & \\
\hline Relaciones con la comunidad & $\bullet$ & & \\
\hline Gestión de asuntos públicos, issues management & & & \\
\hline Patrocinio & & & \\
\hline Publicaciones & $\bullet$ & & \\
\hline Organización de eventos & & & \\
\hline Comunicación con inversores & & \\
\hline
\end{tabular}

Fuente: elaboración propia a partir de cuestionarios auto-administrados 
El cuadro anterior permite apreciar que los departamentos de comunicación no gestionan y controlan todas las funciones tomadas de la literatura científica. Sí que pueden observarse similitudes: en las tres firmas esta área gestiona la comunicación con empleados (comunicación interna) así como las relaciones con los medios de comunicación, ambas funciones tradicionalmente asociadas a los departamentos de comunicación.

Respecto a las diferencias, mientras que Orange integra en su departamento de comunicación las actividades de marketing y publicidad, Telefónica y Vodafone no. Desde Orange se argumenta: “Nosotros en realidad somos intermediarios con los grupos de interés. Yo creo que uno de los principales grupos interés, el core de grupos de interés, es el cliente. Y nosotros somos un intermediario muy importante para él" (Orange). En cambio, en Telefónica las funciones de publicidad y marketing se dirigen desde las unidades de negocio. Desde esta perspectiva, se considera que ambas funciones sirven a las áreas comerciales de la empresa particulares, empresa, digital, etc.- y, por esta razón, dependen de ellas y no de la Dirección de Comunicación Corporativa y Medios. En el caso de Vodafone sucede de manera similar ya que estas funciones son asumidas por un área dedicada exclusivamente al Marketing.

Otra diferencia que se observa es que, si bien todas las empresas coinciden en la relevancia de las relaciones institucionales, sólo en el caso de Vodafone se gestionan desde la Dirección de Comunicación. Telefónica opta por hacerlo desde la Dirección de Asuntos Públicos, también dependiente de Presidencia y en el caso de Orange desde la Dirección de Relaciones Sectoriales, aunque matizan que los asuntos se coordinan con un profesional de comunicación. Esta situación puede explicarse, en parte, por el tipo de sector que se está analizando. La empresa de telecomunicaciones es una entidad altamente regulada, controlada y supervisada. Por eso, estrecha relaciones con distintos organismos, como la Comisión Nacional de los Mercados y de la Competencia, el Consejo Asesor de las Telecomunicaciones y de la Sociedad de la Información, distintas asociaciones sectoriales o la Oficina de Atención al Usuario de Telecomunicaciones. La profusión de agentes, además de la necesidad de contar con unos conocimientos legales especializados, pueden explicar por qué estas relaciones se gestionan desde unos departamentos creados ad hoc y no desde las áreas de comunicación.

Unido a ello, resulta una práctica común contratar empresas especializadas en servicios de comunicación, como por ejemplo, consultoras, agencias de publicidad o de diseño gráfico. Si bien ninguna de las funciones de la tabla están completamente externalizadas en este tipo de empresas externas, sí es cierto que prestan servicios de manera puntual para determinados proyectos como es el caso de la organización de eventos.

En la misma línea, los Patrocinios en el caso de Vodafone y Telefónica son gestionados por otras áreas de la firma, por ejemplo, Responsabilidad Social Corporativa e incluso, en algunos casos, gestionados desde la Fundación de las compañías. Finalmente, la comunicación con inversores es una función asumida por departamentos especializados en las relaciones con este tipo de stakeholdes, por ejemplo, la oficina del accionista. 


\section{Coordinación entre divisiones del departamento}

Por último, se exponen los resultados sobre la coordinación entre las funciones descritas. De acuerdo con el análisis doctrinal, la comunicación es un área cuya complejidad requiere de una administración coordinada y una orientación común de los objetivos.

Las tres direcciones de comunicación del estudio responden positivamente a la pregunta del cuestionario: ¿¿xiste algún tipo de coordinación entre las áreas responsables dentro del departamento?

En el caso de Vodafone, se realizan reuniones esporádicas -sin periodicidad determinada- entre las distintas áreas para elaborar proyectos puntuales y el diseño de planes estratégicos conjuntos. La profesional entrevistada añade: "En empresas multinacionales como la nuestra coordinar toda la comunicación es una tarea compleja pero necesaria para ofrecer una imagen consistente ante nuestros stakeholders" (Vodafone).

También en Telefónica se coordina las áreas de comunicación a través de un comité permanente que se reúne semanalmente en el que, además de elaborar los planes estratégicos de comunicación, se ejecutan las acciones o políticas conjuntas y se elaboran los informes o reportes. En dicho comité participan los directivos y cargos intermedios de cada área dentro del departamento: "El objetivo es comunicar con claridad y consistencia dentro y fuera de las fronteras físicas de la compañía” (Telefónica).

En Orange un comité coordina de manera permanente todas las áreas -Comunicación interna, externa, Publicidad, Marca y Patrocinios-. En él intervienen cargos directivos e intermedios. Así, prácticamente todas las semanas el director de Marca y Comunicación se reúne con los responsables de cada una de las áreas. De esa forma, se informa y se ponen en común las decisiones que afectan a varios o a todos los equipos. Esta responsable apela a la necesaria coordinación de las funciones de comunicación:

"Es muy complicado llevar al exterior un valor si no lo llevas a la práctica dentro (...). Todos los aspectos comunicativos van alineados porque no tendría sentido que los mensajes al exterior no fueran compartidos dentro de la organización" (Orange).

Con estos hallazgos se recogen algunos de los principios apuntados en el marco teórico: la importancia de gestionar todo lo que la empresa dice o hace de manera unitaria y consistente (Christensen et al., 2009).

\subsubsection{Objetivo de investigación 2: director de comunicación y alta dirección}

A continuación, se describe la representación de la unidad de comunicación en los máximos órganos directivos de las empresas. Tal y cómo se ha apuntado desde el análisis doctrinal, la participación de su responsable en la alta dirección es un aspecto clave para valorar el nivel de responsabilidad y poder que se otorga al departamento dentro de las empresas. 
La respuesta acerca del perfil directivo es unánime: todas las operadoras consideran al dircom como el máximo ejecutivo responsable de la comunicación de la corporación y en todas ellas esta figura tiene presencia en el primer nivel del organigrama.

En Vodafone el director de Comunicación y Sostenibilidad forma parte del comité directivo de la compañía. Entre sus funciones más relevantes: representa los asuntos de comunicación en este órgano, participa en iniciativas estratégicas como procesos de adquisición, apertura hacia nuevos mercados, apoyo en el lanzamiento de productos y servicios, etc. Además, asesora de manera habitual a este organismo en materia de comunicación, especialmente en el caso de las declaraciones institucionales. Además, reporta directamente al Presidente de la filial española.

Por su parte, en Telefónica la directora de Comunicación Corporativa y Medios reporta al Presidente. El departamento tiene representación en el comité directivo pero "además de representar al departamento esta figura participa activamente en iniciativas estratégicas de la empresa, por lo tanto, toma parte en las decisiones al más alto nivel” (Telefónica). Entre sus cometidos destacan: asesorar al Comité Directivo en materia de comunicación -declaraciones institucionales, gestión de relaciones-y representar los asuntos de comunicación.

En Orange el director general de Comunicación y Marca también forma parte del comité de dirección de la compañía. Según afirman, que el dircom tenga una posición tan cercana al Consejero Delegado en España es uno de los grandes valores de la empresa, de hecho, reporta a esta figura. Además, existe una coordinación directa con la Dirección de Comunicación de la matriz para alinear la comunicación de todo el grupo.

En las tres firmas, el director de comunicación es un miembro de la alta dirección y cargo de alto gobierno empresarial. Todos ellos tienen unas responsabilidades que son consideradas “críticas”. Entre ellas, destacan dos de forma unánime. Por un lado, el asesoramiento continúo en materia de comunicación y, por otro, la representación de los asuntos de comunicación claves ante el comité directivo.

La integración del dircom en la alta dirección queda patente en los resultados de la encuesta realizada y se confirma en las entrevistas. "Si los directores de comunicación no están plenamente implicados en el equipo directivo difícilmente podrán hacer una contribución directa” (Orange).

Esta implicación puede observarse teniendo en cuenta tres indicadores. De entrada, todos los dircom del estudio son considerados miembros de la alta dirección. En otras palabras, tienen influencia en la estructura directiva de las operadoras. En segundo lugar, dependen directamente del primer nivel ejecutivo, ya sea Presidente o Consejero Delegado, según sea el caso. En tercer lugar, participan activamente en el comité de dirección y, por tanto, en la toma de decisiones estratégicas. Respecto a este punto y, en coherencia con lo apuntado en la revisión teórica, los tres responsables de comunicación matizan la diferencia, nada baladí, entre participar activamente en las decisiones estratégicas de la empresa -procesos de 
adquisición, nuevos mercados, lanzamiento de productos y servicios, etc.- y simplemente rendir cuentas sin participar en el debate y discusión sobre estos asuntos.

En los tres casos analizados la figura del dircom se considera fundamental en la toma de decisiones de este órgano. De este modo, "participa en todos los planes decisivos de la compañía desde su origen hasta la implementación" (Vodafone). En estas firmas queda patente la importancia de la comunicación, reflejada en el estatus elevado de su director en el organigrama organizativo.

\subsubsection{Objetivo de investigación 3: coordinación con otras divisiones estratégicas}

Trazar puentes con el resto de áreas de la empresa es un tema recogido en líneas anteriores. Cornelissen (2004) argumenta que este tipo de gestión provoca que las fronteras entre el centro corporativo de las empresas, las distintas unidades de negocio y la unidad de comunicación sean más porosas y permeables.

Las tres operadoras estudiadas consideran que la función de comunicación requiere de una gestión transversal y, para ello, la coordinación se perfila como una "necesidad" en organizaciones tan complejas. Dichas firmas, son multinacionales y, por tanto, requieren de flujos de comunicación continuos entre la empresa matriz y las filiales así como la coordinación entre el departamento de comunicación y el resto de áreas.

Con respecto a la coordinación entre filiales y matriz, explican: "Hay una coordinación directa con la Dirección General de Comunicación del grupo porque la comunicación debe estar alineada, en muchos aspectos los principios son comunes para todo el grupo aunque luego haya que “localizar" según la filial” (Orange).

Igualmente, la coordinación entre departamentos se perfila como un pilar de gestión clave. "El departamento de comunicación no puede actuar en solitario sino que presta servicio al resto de áreas" (Vodafone). En el caso de esta empresa se coordinan con otras áreas como Marketing, Recursos Humanos, RSC, Legal, Unidades de Negocio, Servicio al Cliente e I+D. Gracias a un comité permanente su responsable se reúne de manera continua con estos departamentos, además de realizar reuniones esporádicas. En ellos participan directivos y cargos intermedios de cada una de estas áreas. Trabajan fundamentalmente en proyectos conjuntos y en la elaboración de planes estratégicos de la compañía.

También la Dirección de Comunicación Corporativa y Medios de Telefónica "tiende puentes de manera constante entre las distintas áreas de la empresa así como entre la matriz española y sus filiales en el resto del mundo” (Telefónica). El departamento establece mecanismos de coordinación con las áreas de Marketing, Recursos Humanos, RSC, Legal, Unidades de Negocio, Servicio al Cliente e I+D donde intervienen cargos intermedios pero también directivos -exceptuando I+D-. Existe un comité específico permanente con el área de Recursos Humanos, Unidades de Negocio y Servicio al Cliente donde se elaboran planes estratégicos conjuntos. Unido a ello, se reúnen de manera esporádica con Marketing, RSC, legal 
e I+D, con cuyos responsables realizan proyectos ocasionales. También ejecutan acciones o políticas conjuntas con Marketing, RSC, Recursos Humanos, Unidades de Negocio y Servicio al Cliente. Con estas tres últimas áreas también elaboran informes o reportes conjuntos que se envían al comité directivo.

En Orange Comunicación y Marca mantiene una coordinación con las áreas de Recursos Humanos, Servicio al Cliente, RSC, Legal y con las Unidades de Negocio. Con tal fin, emplean comités permanentes, reuniones, informes, diseño de estudios conjuntos que aportan valiosa información al departamento de comunicación. Sobre las unidades de negocio especifican:

“Nos coordinamos con ellos en aspectos de la comunicación que les afectan como, por ejemplo, la comunicación de un producto o un proyecto nuevos. Las áreas de negocio o técnicas son las expertas en su campo y para nosotros sería imposible trabajar sin la información que nos aportan, aparte de que Comunicación es un área de soporte a su trabajo" (Orange).

De esta manera, emplean comités permanentes, reuniones, informes, diseño de estudios conjuntos que aportan valiosa información al departamento de comunicación.

Si bien cada operadora estudiada emplea distintas herramientas de coordinación y la frecuencia en la interacción varía, en todas ellas la transversalidad comunicativa es un imperativo. Entre los sistemas empleados destacan los comités permanentes, las reuniones periódicas y la información compartida entre el departamento de comunicación y el resto de áreas.

Los anteriores hallazgos conducen a la siguiente reflexión: para hacer efectivas las relaciones entre las matrices y las distintas filiales así como en las relaciones interdepartamentales, la comunicación adquiere una dimensión clave.

\section{Discusión}

Las respuestas expuestas confirman la hipótesis planteada: las tres empresas de la muestra realizan una gestión estratégica de su comunicación en un entorno multistakeholder. Concretamente revelan que la gestión de la comunicación en empresas de telecomunicaciones es una tarea altamente compleja. La internacionalización o el impacto tecnológico y digital son factores que las han transformado. Estrategia e inmediatez son dos realidades que conviven en este mercado, difíciles de compaginar. Esto requiere una dinámica de trabajo ágil y flexible para abordar grandes proyectos a medio y largo plazo.

Para afrontar semejantes retos la comunicación se perfila como un aliado estratégico en el presente y futuro de las organizaciones. De este modo, los resultados reflejan el cambio acelerado que vive el rol de la comunicación en estas firmas, acelerado por las exigencias sociales y de mercado. Ésta ha evolucionado hacia formas más complejas y estratégicas. Una 
auténtica revolución que ha modificado la forma en que las empresas se relacionan con los distintos stakeholders y en la que el dircom y su equipo constituyen un soporte fundamental.

Las direcciones de estas empresas son conscientes de esta realidad y, por esta razón, los departamentos de comunicación están dotados de una estructura organizativa a nivel corporativo y con una ubicación en el más alto nivel. No se trata de un aspecto meramente formal, sino de dar soporte y recursos a un departamento cuyas funciones van mucho más de allá de un carácter técnico, táctico y operativo. Este hecho deja entrever que la comunicación como función es valorada internamente, su director es una figura reconocida en la gestión estratégica y, además, tiene una influencia directa en la toma de decisiones de sus empresas. Las respuestas ponen de manifiesto la relevancia de la dimensión estratégica de este departamento con varias implicaciones en su gestión.

En primer lugar, los profesionales entrevistados subrayan la importancia de la orientación al negocio, los resultados y la aportación de valor. En la literatura es un lugar común describir los objetivos estratégicos empresariales como las semillas de las que nacen a posteriori las políticas comunicativas. Sin embargo, esta sucesión temporal ha dejado de tener sentido en la actualidad, dado que el dircom, en empresas como las estudiadas, se implica directamente en la elaboración y diseño de los planes estratégicos desde su origen hasta su implementación. Y para que esta aportación sea realmente valiosa se hace necesario que esta figura equilibre las dimensiones institucionales y comerciales como ningún otro directivo en la empresa.

En segundo lugar y respecto a las funciones, la academia explica que el departamento de comunicación gestiona todas las funciones de comunicación apuntadas en el cuadro 7. Sin embargo, cabe matizar que ninguna de las direcciones de comunicación de la muestra gestiona por completo todas las funciones de comunicación, ya que en algunos casos, tales funciones dependen de otras áreas de la empresa.

Con todo, los responsables entrevistados afirman que, más allá de dirigir, gestionar y controlar el presupuesto de todas y cada una de las actividades de comunicación, lo verdaderamente importante es contar con instrumentos internos que les permita coordinar y obtener información de otras direcciones. Esto implica que en las empresas departamentos o funciones como Comunicación corporativa, Marca, Publicidad, Dirección comercial, etc, tendrán que aprender a hablar el mismo lenguaje, a trabajar juntos y orientarse hacia objetivos conjuntos. La integración es la base sobre la que se construye una aproximación holística al complejo fenómeno de la comunicación en la empresa.

Finalmente, la estrategia de comunicación puede ser creada desde los departamentos de comunicación pero sólo tendrá impacto si se implica en su ejecución a todos los líderes y áreas de la compañía. Los entrevistados ponen de manifiesto la necesidad de integrarse en equipos multidisciplinares con departamentos como Recursos Humanos, Unidades de Negocio o Innovación. Según explican, estos procedimientos les facilita una óptica más amplia desde distintos ángulos, aspectos clave para el correcto funcionamiento de un departamento como el de comunicación. 
Dado que se asume que la comunicación es un elemento transversal en la organización, describir la actividad de este departamento se antoja insuficiente si se ignoran los procesos comunicativos que se dan de modo inherente en el transcurso de las decisiones sobre políticas corporativas y en el trabajo en equipos. De ahí que sea relevante el análisis de cómo el departamento tiende puentes con otras áreas estratégicas para las operadoras.

La tercera parte del estudio revela cómo la comunicación en la empresa no se reduce a la información ofrecida por las personas que coordinan el departamento de comunicación. Existen otro tipo de relaciones comunicativas que, aunque no sean gestionadas directamente por la unidad de comunicación, sí se producen como consecuencia de la riqueza del fenómeno comunicativo como un aspecto transversal en las organizaciones.

\section{Conclusiones}

La comunicación es un concepto rico en constante evolución. Esto explica por qué reducir su función a transmitir o difundir mensajes se antoja más que insuficiente. Unido a ello, la comunicación comercial dirigida al consumidor y destinada a vender productos y servicios, aun siendo fundamental y estratégica para el negocio, no tiene capacidad para responder, por sí sola, a todas las demandas comunicativas de un ambiente multistakeholder, 24 horas, los siete días de la semana.

Tampoco el enfoque táctico, centrado en programas de comunicación destinados a generar notoriedad entre los medios, estará a la altura de la complejidad que supone gestionar un elemento que soporta el cultivo de preciados recursos intangibles como: conocimiento, confianza, reputación, lealtad o los valores corporativos. Todos ellos inimitables desde una perspectiva competitiva a largo plazo.

Los retos a los que nos enfrentamos demandan un tipo de gestión comunicativa multistakeholder, directiva y transversal.

- Multistakeholder: los grupos de interés reclaman como mínimo ser escuchados por las empresas, cuando no consultados, e incluso participar en procesos de decisión que les atañe. Si la forma de relacionarse con éstos se ha transformado también será necesario redefinir el papel de la dirección de comunicación. Grupos de interés tan diversos como accionistas, clientes, proveedores, empleados u ONGs por mencionar algunos pueden tener demandas y expectativas diferentes e, incluso, contradictorias respecto a la actividad de la empresa. Así pues, el profesional de comunicación se presenta como un mediador imprescindible capaz de conectar a los líderes empresariales con el contexto social y ser nexo de unión.

Desde este punto de vista, una buena gestión de las relaciones con el entorno puede otorgar a los directivos pautas para responder a las demandas de sus grupos de interés, dar respuesta a los porqués de las decisiones adoptadas y cultivar relaciones basadas en la transparencia y la prudencia. Así, el profesional tendrá que estar atento a esta compleja realidad, tener una visión amplia, capacidad de escucha y de respuesta. Un modelo de comunicación mucho más complejo pero, sin duda, más rico que el unidireccional centrado en ganar una notoriedad no siempre necesaria ni deseable. Y precisa- 
mente, será en ese punto donde sus profesionales demostrarán su verdadera aportación estratégica y transformadora en las organizaciones.

- Directiva: la comunicación es una variable más que deben considerar los altos directivos en su gestión y procesos de toma de decisiones. Si la gestión de la información tiene una importancia crítica, el acceso directo a los profesionales clave de la compañía será vital para que el dircom pueda hacer adecuadamente su trabajo y logre diseñar un plan de comunicación que aporte a los objetivos generales de negocio.

Estas variables han sido tradicionalmente un anhelo de los dircom y ha sido cumplido, al menos, en empresas multinacionales como las estudiadas. Aun así, todavía queda un largo camino para situar la comunicación en los centros de decisión de las compañías del mismo modo que la dirección jurídica o financiera, por poner sólo dos ejemplos. De este modo lo testifican algunos informes del estado de la profesión donde directores y ejecutivos del área de comunicación reconocen ciertas lagunas en su formación. Concretamente, en aspectos relacionados con la gestión y estrategia empresarial y en disciplinas como las finanzas, la dirección de personas o el liderazgo.

El futuro pasa por mejorar la formación y capacitación de estos profesionales. Se dará en la diana si se atienden dos aspectos concretos: primero, aumentar los conocimientos en gestión para integrarse en equipos directivos y, segundo, la necesidad de trabajar en métricas e indicadores clave (KPI) que puedan integrarse en el cuadro de mando integral de las firmas. Estos retos son de tal envergadura que la comunidad científica y profesional tendrán que caminar de la mano para detectar líneas de mejora, implantarlas, estudiarlas y trabajar sobre las mejores prácticas.

- Transversalidad: la comunicación lejos de ser considerada una labor exclusiva de un conjunto de profesionales acotados en un departamento, traspasa sus fronteras departamentales y requiere de una coordinación con el resto de áreas de la compañía. Este hecho no esconde la necesidad de liderarla con el departamento de comunicación y su responsable al frente. Desde ahí, se administrarán los tiempos pertinentes, la necesaria continuidad y consistencia en los mensajes o principios como la claridad informativa.

Este panorama dibuja nuevas rutinas de colaboración donde los profesionales de comunicación tendrán necesariamente que salir de sus áreas y desarrollar una enorme capacidad de interacción con otros departamentos y áreas de la empresa. Dado que la comunicación es, como se ha mencionado, una realidad porosa en las organizaciones, las rutinas y mentalidad de los profesionales también tendrán que serlo. Valores y capacidades como el diálogo, la confianza, participación y cooperación serán altamente valorados para el ejercicio de esta profesión. Y, al mismo tiempo, el resto de áreas tendrán que ser conscientes de la dimensión comunicativa de su labor y de la relevancia de los tan mencionados intangibles (reputación, innovación, responsabilidad social, derechos de autor, etc) cuya gestión tiene resultados tan tangibles como los relacionados con la cotización, el consumo de productos o el éxito de los proyectos empresariales. 


\section{Referencias bibliográficas}

Argenti, P. (2014): Comunicación estratégica. Madrid: LID.

ArthurW. Page Society, (2007): The authentic enterprise. Disponible en: http://www.awpagesociety.com/wp-content/uploads/2011/09/2007AuthenticEnterprise.pdf. [Consultado el 30/04/2014].

Berger, B. K. y Meng, J. (eds.) (2014): Public relations leaders as sensemakers. A global study of public relations and communication management. Nueva York: Routledge.

Blay, R. y Benlloch, M. T. y Sanahuja, G. (2014): "Análisis de la gestión de la comunicación en los sectores industriales tradicionales valencianos”, Doxa Comunicación, n. 18, pp. 55-82.

Brønn, P. S. (2014): “How others see us: leaders' perceptions of communication and communication managers”, Journal of Communication Management, vol. 18, n. 1, pp. 58-79.

Caballero, M. A. (2002): La comunicación institucional como instrumento estratégico para el logro de los objetivos de las organizaciones. Estudio del Plan de Comunicación del Programa TIES en Telefónica, Tesis doctoral inédita presentada en la Facultad de Comunicación de Universidad de Navarra.

Cambra, J., Melero, I. y Sese, F. J. (2012): “Aproximación al concepto de engagement: un studio exploratorio en el sector de la telefonía móvil”, Universia Business Review, n. 33, pp. 84-103

Christensen, L. T., Morsing, M. y Cheney, G. (2008): Corporate Communications: Convention, Complexity, and Critique. Londres: Sage.

Comisión Nacional de los Mercados y de la Competencia (2017): Informe Económico de las Telecomunicaciones y del sector audiovisual 2016. Disponible en: http://data.cnmc.es/datagraph/files/Informe\%20Telecos\%20y\%20Audiovisual\% 202016.pdf. [Consultado el 10/03/2017].

Cornelissen, J; van Bekkum, T. y van Ruler, B. (2006): “Corporate communications: A practice based theoretical conceptualization”, Corporate Reputation Review, n. 9(2), pp. 114-133.

Cornelissen, J. y Lock, A. R. (2001): “The appeal of integration: managing communications in modern organizations”, Marketing Intelligence \& Planning, vol. 19, n. 6, pp. 425-431.

Cornelissen, J. y Thorpe, R. (2001): “The organisation of external communication disciplines in UK companies: a conceptual and empirical analysis of dimensions and determinants", The Journal of Business Communication, vol. 38, n. 3, pp. 413-438.

Costa, J. (2011): Identidad corporativa. Mexico: Trillas.

Denzin, N. K. y Lincoln, Y. S. (1994): Handbook of qualitative research. Thousand Oaks: Sage.

DIRCOM (2016): Anuario de la comunicación 2016. Madrid: Asociación de Directivos de Comunicación. 
DIRCOM (2015): Anuario de la comunicación 2015. Madrid: Asociación de Directivos de Comunicación.

DIRCOM (2014): Anuario de la comunicación 2014. Madrid: Asociación de Directivos de Comunicación.

DIRCOM (2010): "El estado de la comunicación en España 2010". Disponible en: http://www.dircom.org/images/stories/news/Noticias/ActualidadDircom/presentacionestadodelacomunicacione\%20espaa2010.pdf

DICRCOM (2005): “El estado de la comunicación en España 2005”. Disponible en: http://www.dircom.org/publicaciones/estudios/item/549-el-estado-de-la-comunicaci\%C3\%B3n-en-espa\%C3\%Bla-2005

Falkheimer, J; Heide, M., Simonsson,C; Zerfass, A.; Verhoeven, P. (2016): "Doing the right things or doing things right? Paradoxes and Swedish communication professionals' roles and challenges", Corporate Communications: An International Journal, 21(2), pp. 142-159.

Falkheimer, J. (2014): “The power of strategic communication in organizational development”, International Journal of Quality and Service Sciences, vol. 6, n. 2/3, pp. 124-133.

Frandsen, F. y Johansson, W. (2015): “The role of communication executives in strategy and strategizing”, en Holtzhausen, D. y Zerfass, A. (eds.): The Routledge handbook of strategic communication. Nueva York: Routledge, pp. 229-243.

García de los Salmones, M. S. (2001): La imagen de la empresa como factor determinante en la elección del operador: identidad y posicionamiento de las empresas de comunicación móviles. Tesis doctoral inédita presentada en la Universidad de Cantabria.

González, C. y Monleón, P. (2013): “La gestión de intangibles en la Dirección de Comunicación Corporativa. Estudio sobre el DirCom en las organizaciones del Reino Unido", Doxa Comunicación, n. 17, pp. 27-56.

Gregory, A. (2011): "The state of the public relations profession in the UK: A review of the first decade of the twenty-first century", Corporate Communications: An International Journal, vol. 16, n. 2, pp. 89-104.

Gregory, A. y Willis, P. (2013): Strategic public relations leadership. London: Routledge.

Grunig, L. A; Grunig, J. E. y Dozier, D. M. (2002): Excellent public relations and effective organizations, A study of communication management in three countries. Mahwah: Lawrence Erlbaum Associates.

Gutiérrez-García, E. (2014): "El dircom, cartógrafo social y estratega en las instituciones”, en Gutiérrez-García, E. y Rodríguez Virgili, J. (eds.): El futuro de la comunicación. Madrid: LID, pp. 25-36.

Gutiérrez-García, E. y Recalde, M. (2016): “Dialogue for strategic decision-making processes: an IDEA model”, en Brønn, P. S., Romenti, S., y Zerfass, A. (eds.): The Management Game of Communication. Advances in Public Relations and Communication Management. Bruselas: Emerald Group Publishing Limited, pp. 245-263.

Hallahan, K; Holtzhausen, D; van Ruler, B; Verčič, D. y Sriramesh, K. (2007): "Defining strategic communication”, International Journal of Strategic Communication, vol. 1, n. 1, pp. 3-35. 
Herranz de la Casa, J. M. (2007): “La comunicación en las organizaciones de la economía social”, en Salinas, F., Herranz de la Casa, J. M. (eds.): Empresas de economía social: aspectos para su desarrollo. Ávila: Universidad Católica de Ávila, pp. 6196.

Johansson, C. y Ottesting, A. T. (2011): “Communication executives in a changing world: legitimacy beyond organizational borders”, Journal of Communication Management, vol. 15, n. 2, pp. 144-164.

Losada Vázquez, A. (1998): La comunicación institucional en la gestión del cambio: el modelo universitario. Salamanca: Universidad Pontificia de Salamanca.

Maxwell, J. A. (1996): Qualitative research design. An interactive approach. California: Sage.

Miquel, S. y Navarro, M. (2016): "Análisis de la investigación sobre la historia y evolución de las relaciones públicas y la comunicación corporativa en España”, Doxa Comunicación, n. 22, pp. 129-146.

Moss, D; Warnaby, G. y Newman, A. J. (2000): "Public relations practitioner role enactment at the senior management level within UK companies”, Journal of Public Relations Research, vol. 12, n. 4, pp. 277-307.

Patton, M. Q. (1990): Qualitative evaluation and research methods. Londres: Sage.

Raupp, J. y Hoffjann, O. (2012): “Understanding strategy in communication management”, Journal of Communication Management, vol. 16, n. 2, pp. 146-161.

Recalde, M. y Gutiérrez-García, E. (2015): “La comunicación en el tablero de decisiones en la empresa: Algunas aportaciones estratégicas”, Anàlisi. Quaderns de Comunicació i Cultura, n. 52, pp. 97-112.

Swerling, J. y Sen, C. (2009): “The institutionalization of the strategic communication function in the United States", International Journal of Strategic Communication, vol. 3, n. 2, pp. 131-146.

Van Riel, C. B. M. (2012): Alinear para ganar. Valencia: LID Editorial Empresarial.

- (1997): “Research in corporate communications: overview of an emerging field”, Management Communication Quarterly, vol 11, n. 2, pp. 288-309.

Walesska, M. S. D.y Alvarado, A. H. (2009): “Imagen y reputación corporativa. Estudio empírico de operadoras de telefonía en España”, Teoría y Praxis, n. 6, pp. 9-29.

Wang, A. (2009): “Perceptions of Corporate Social Responsibility practices on mobile phone companies”, International Journal of Mobile Marketing, vol. 4, n. 1, pp. 62-68.

Zerfass, A; Tench, R;Vercic, D., Verhoeven, Moreno, A; Tench, R; Vercic, D. (2016): “European Communication Monitor 2016. Exploring trends in big data, stakeholder engagement and strategic communication. Results of a survey in 43 countries”. Disponible en: http://www.zerfass.de/ECM-WEBSITE/media/ECM2016-Results-ChartVersion.pdf. [Consultado el $15 / 08 / 2016]$. 